\title{
Sign Language Translator Using YOLO Algorithm
}

\author{
Bhavadharshini $\mathrm{M}^{\mathrm{a}, 1}$, Josephine Racheal $\mathrm{J}^{\mathrm{a}}$, Kamali $\mathrm{M}^{\mathrm{a}}$, Sankar $\mathrm{S}^{\mathrm{b}}$ and \\ Bhavadharshini $\mathrm{M}^{\mathrm{b}}$ \\ a Student, Department of Computer Science, KCG College of Technology, Chennai. \\ ${ }^{\mathrm{b}}$ Professor, Department of Computer Science, KCG College of Technology, Chennai.
}

\begin{abstract}
Sign language is a terminology that encloses a motion of hand gestures which is an environment for the auditory impairment, individual (deaf or dumb) to deal with others. Nevertheless, so as to impart with the hearing impaired individual, the communicator obtains to acquire acquaintance in sign language. As follows is frequent to make undoubted that the message provided by the hearing impaired person acknowledged. This implemented system propounds an implementation of real time American Sign Language perception in Convolutional Neural Network (CNN) with the support of You Only Look Once version (YOLO) algorithm. The algorithm initially executes data acquisition, subsequently the pre-processing of gestures and are conducted to trace hand movement utilize a combinational algorithm.
\end{abstract}

Keywords. Sign Language Translation, Convolutional Neural Network (CNN), YOLO algorithm, hand tracking, segmentation, American Sign Language (ASL).

\section{Introduction}

Communicating is the mode of trading knowledge between transmitter and receiver via any environment accessible. The perception among two parties is key to assure that the message communicated is substantially deciphered by the receiver. Hard of hearing individuals utilize sign dialect as an environment to impart with rest. Sign dialect is the course of communication that's determined on hand development and optical orientation. Sign dialect specialists indicated that the visual accustomed sustains of hand shape (the way the hand and fingers shape a sign), an area of the hand, palm introduction and progress of the hand as its high spots. These profess illuminates that each hand signal or development encompasses distinctive significance to be mapped.

This study could be a CNN-based human hand signal recognition methodology [1]. CNN could be an investigate section of neural networks. Application of CNN to memorize human signals, there's no need to create complicated calculations to extricate picture features and determine them [2]. With the help of the convolution and sub-sampling level of a CNN, invariant highlights are permitted with little disruption. To decline the collision of different hand postures of a hand signal sort on the acknowledgment preciseness, the principal axis of the hand is found to calibrate the picture in this work [3]. Calibrated

\footnotetext{
${ }^{1}$ Bhavadharshini M, Department of Computer Science, KCG College of Technology, Chennai, India.

E-mail: bhavimurugan16101999@gmail.com
} 
pictures are profitable to a $\mathrm{CNN}$ to memorize and recognize precisely. In a genuine circumstance, when ordinary individuals encounter with deaf people, communication deterioration arises due to different manners of communication. In order to convey with the hearing disabled individual, the information about sign dialect is indispensable, merely it fetch to be an obstruction for those who don't learn the dialect. The most common limitation confronted by hard of hearing individuals in communication is the nonattendance of a flag mediator [4]. Individuals are not keen to memorize sign dialect on account of the miserable stipulate of sign dialect course for ordinary individuals.

\section{Literature Survey}

The author of [5] conducted a framework entitled as "Indian sign language translator using gesture recognition algorithm”. The framework interprets motions made in ISL into English. The gesture acknowledgment framework is to ensure gestural information. Vision based strategy incorporates picture refinement. The database for creating this framework is made to possess with the recorded recordings of hard of hearing and quiet endorsers. This makes the signals included to be authentic. The diversity of different calculations for Pre-processing, Feature extraction and vector quantization, the leading skillful calculation was shortlisted to be a combined yield calculation for pre-processing, 2D FFT Fourier Descriptors for feature extraction and 4 vector codebook LBG. The authors of [6] proposed a convolutional neural network (CNN) strategy for recognizing hand signals from camera activities of human task exercises. To obtain the CNN's preparing and examining the details, the skin demonstration and the gauging of hand location and introduction are related. Since light conditions have a major impact on complexion color, the proposed utilize a Gaussian Mixture model (GMM) to gear up the skin exposure; the latter is employed to effectively filter out non-skin color in an illustration. The contemplated framework has also acquired the palatable comes about on the attributive motions in an unrelenting movement utilizing the contemplated guideline. The authors of [7] discussed a sign dialect acknowledgment framework utilizing Back Propagation Neural Network Calculation contemplated instituted on American Sign Language. The proposed framework employments the pictures in accordance with the nearby framework or the outline detained from webcam as an input. The framework employments two classifiers: one employments crude image attributes and the other one employments thresholding highlights. Back propagation Algorithm was utilized for the proposed system as learning assessment. Marcel Inactive Hand Pose was used for the framework as a database.

\section{System Design}

In order to organize a communication with the hearing impaired individual, the communicator should have knowledge in sign vocabulary [8]. The interpretation amongst two parties is exceptionally imperative to secure that the message conveyed is substantially translated by the recipient. In a genuine circumstance, when ordinary individuals encounter with deaf people, disclosure deterioration arises scheduled to different styles of communication. ASL requires utilize of a person's hands so in case something happens where a wrist was sprained and it debilitates that individual from talking [9]. Sign di- 


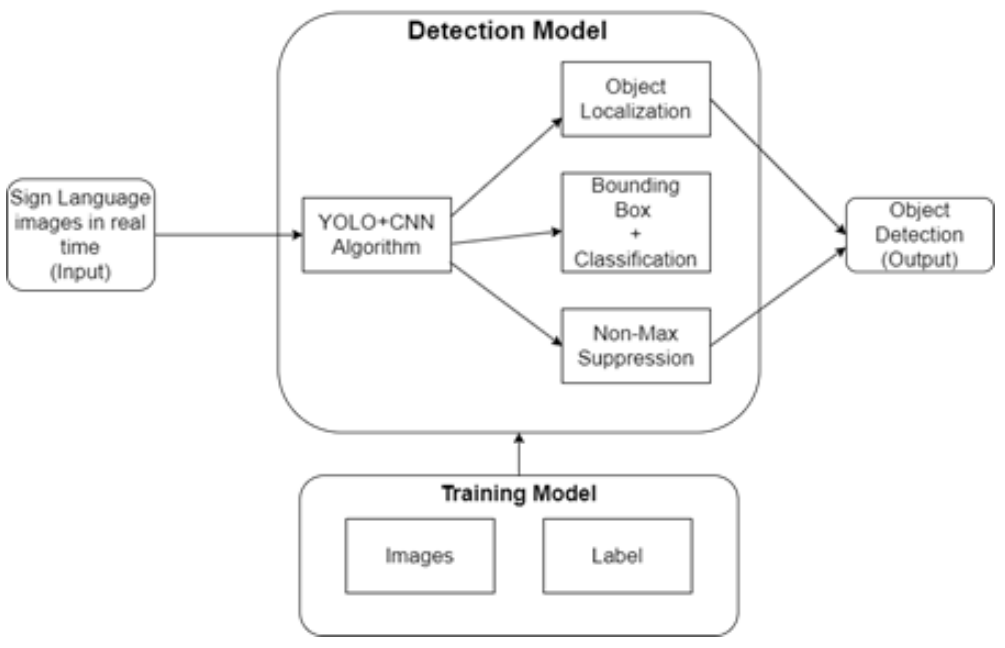

Figure 1. System Design

alect deciphering is one of the highest-risk callings for ergonomic injury. The investigate shows that translating causes more physical stretch to the limits than high-risk errands conducted in mechanical settings, counting gathering line work [10]. It is to found a coordinate interface between an increment within the mental and cognitive push of the interpreter and an increment within the hazard of musculoskeletal wounds such as carpal burrow disorder and tendonitis [11]. In order to solve this problem, the proposed system use Convolutional Neutral Network (CNN) and You Only Look Once (YOLO) algorithm, whereas CNN could be a study branch neural networks [12]. Application a CNN to memorize human movement, there's no necessity to formulate complicated assessment to extricate picture attributes and determine them [13]. CNN fundamentally utilized to perform image classification, protest acknowledgment and question discovery in today's innovation. The accuracy of system increases while using YOLO calculation appears to be one of speediest picture handling calculation with a speed of 45 frames/second, which has high robustness and precision for detecting the American Sign Language. Calibrated pictures are beneficial to a $\mathrm{CNN}$ to memorize and recognize accurately.

\subsection{Methodology}

The framework plan of this venture is as appeared in Figure 1, it was isolated into Training paradigm and Detection paradigm, where the Training paradigm comprises of names and trained database, and the Perception demonstrate as the testing handles of this venture. The framework will start to operate by getting input of sign dialect picture in genuine time via webcam. YOLO assessment will deal the picture by distinguishing the presence of trained snapshots within the input snapshot. On the off chance that the prepared picture exists in the input, a bounding box with a name that focuses the expected object will be compiled. Amid the information compilation of this extend, the sign language dataset from an online source, a database that mostly comprises of commonly used phrase of American Sign Dialect pictures were aggregate. Respectively sign dialect comprises of 500 pictures of $400 \times 400$ determinations with a diversity of radiance. As the 
literacy of American Sign Dialect is mostly known, in this manner a few of the phrases can be straightforwardly utilized in this extend. From the database, the category of sign dialect pictures furthermore partitioned into two sorts, which were remarked as true label and predicted label.

In this setting, inactive sign dialect make reference to a solitary hand progression and different hand progression was determined as an energetic sign dialect [14]. The example of picture naming that was driven amid the database planning. This extends centered on the progression of the inactive sign dialect because it is simpler to be dominated contrast to the energetic sign dialect. Subsequently the pictures were composed, the pictures were named based on the required phrases those signs represent. The annotated pictures data that contain facilitates of the picture was put away within the content directory and will be conducted within the draft session. Afterwards the planning of the database accom-

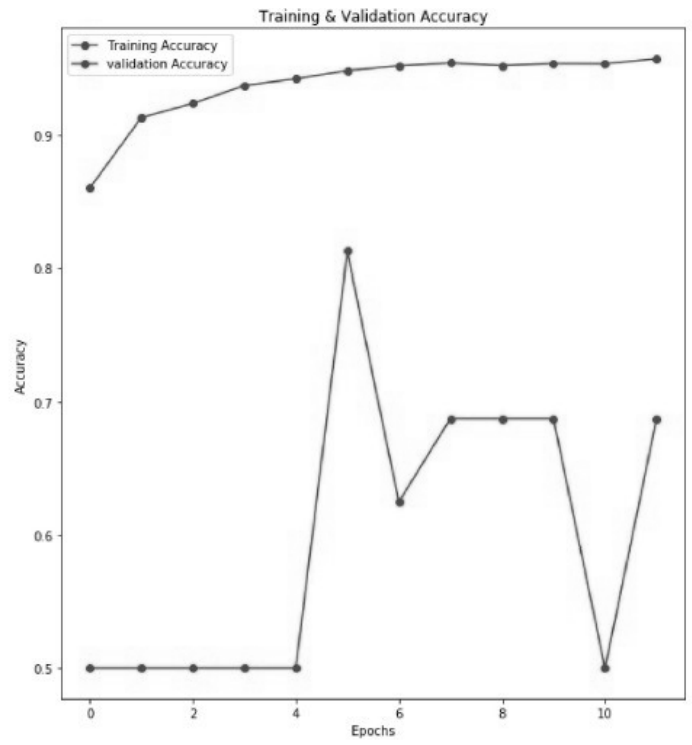

Figure 2. Epochs Vs Validation Accuracy Graph for all the Trained on Required Phrases

plished, the classified perception is grouped accordingly. The training sets are prepared, the desired criterion such as the count of classes and channels were given as parameters in the Python code accordingly. Ensuing, the name log, which embraces a directory of the objects to be entitled, was accomplished. In addition, the training prepare was enforced utilizing convolutional weights and being compiled within the Convolutional Neutral Network $(\mathrm{CNN})$ system. Figure 2 appears the training case that was implemented. Later in the training procedure of demonstrating completed, the model testing prepare was enforced to conclude the precision of the prepared mass [15]. In Figure 3 shows the hand gesture for "sorry" which is extracted from the camera [16]. Later on the most exact weights was distinguished, the weights were analyzed in real-time, by means of which the video was detained utilizing a webcam. The outcomes of the further weights were organized and will be referred about within another segment.

The YOLO (You Only Look Once) algorithm takes the whole picture in a single occasion and predicts the bounding box arranges and class probabilities for these boxes. 


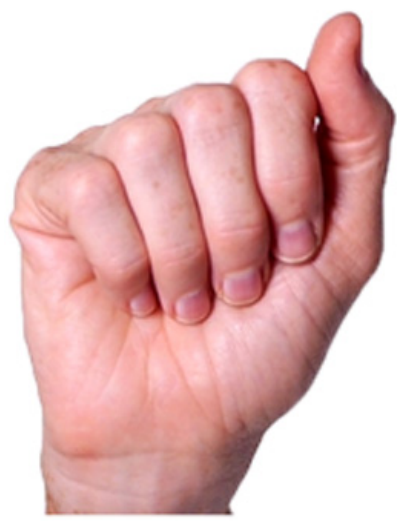

Figure 3. "sorry" in American Sign Language

Image classification and localization are connected on each framework. YOLO at that point predicts the bounding boxes and their comparing class probabilities for objects as seen within the underneath diagram. Convolutional Neural Network (CNN) could be an acknowledgment calculation which utilized in acknowledgment of pictures, sound, content, etc. It has numerous highlights like convolution, pooling, dropout which are a few methodologies utilized to progress fault tolerances.
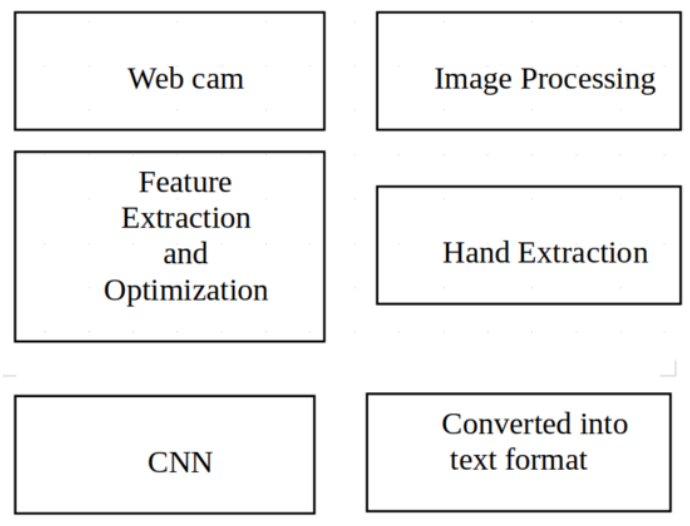

Figure 4. Architecture Diagram for Sign Language Translator

\subsection{Pre-processing}

The pre-processing process initiate with a dataset interpretation where the perception were aggregate and dominated to sort a dataset whichever will be moreover employed for validation and test batch. The perception in the local apparatus or the frame detained from the webcam is employed as input to the technique. Afterwards refinement input image, then classifiers catalogue the image which class it affiliate to accordingly. The Figure 4 shows architecture diagram of sign language translator. 


\subsection{Hand Extraction}

Tracking of a hand is more often than not troublesome as the development of hands can be exceptionally quick and their appearance can alter immensely inside some frames. In a vision schema, the luminance requirement is a substantial constituent to sway the framework execution. Utilizing colour verge to catalogue skin and non-skin colours is prevalent in habitual access; merely colour limits are not adequate to depict the empirical attribute of skin colour under various luminance conditions. Indeed, in spite of the fact that the $\mathrm{YCbCr}$ colour interval that is less delicate to the luminance state than the RGB colour space is deployed, the consequence is still lacking. The Grey Scale pictures for signal acknowledgment are utilized as the acknowledgment rate is moved forward compared to others.

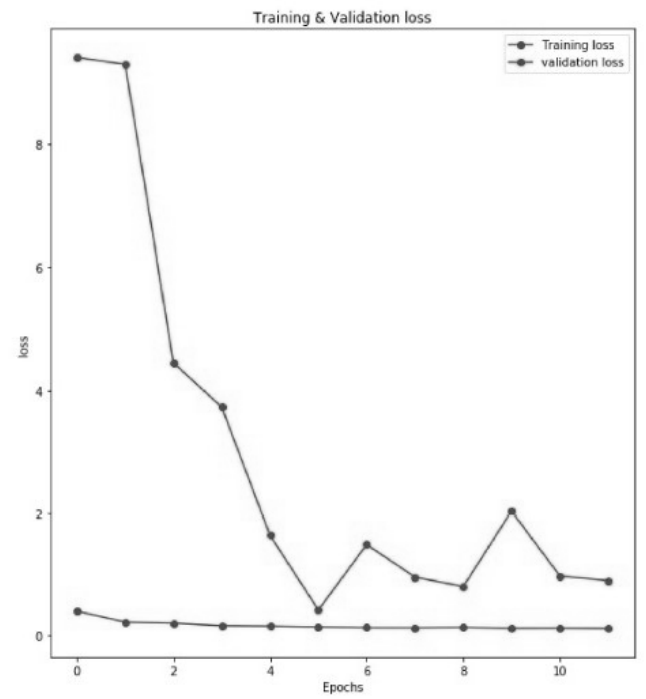

Figure 5. Epochs Vs Validation Accuracy Graph for Models Trained on each phrase

\subsection{Feature Extraction}

Feature extraction cites to the method of recognizing the principal considerations in a picture (intrigued focuses) that can offer assistance to characterize the images substance such as bend, pattern, boundary, and spot. Feature extraction upon the pictures has fetched in this way: Input pictures into the framework are changing over to an encrypted organize which suggests changing over a piece picture into a progression of RGB pixels [17]. YOLO process will prepare input pictures by recognizing the presence of trained pictures. It encompasses the outcome of the anticipated protest within bounding boxes. At that point, pictures are consolidated to be in the corresponding figure. Respectively perception diminishes to 76 x $66 \mathrm{PX}$. 


\subsection{Testing phase}

To begin with, the picture information is perused successively. In conjunction with this, the name records are stacked and the carriage return is stripped off. Following, the file representing the unused prepared demonstration is stacked as a graph in Figure 5. Once the show is stacked, the picture information is stacked as input to the chart, for a preparatory expectation. As a result of this preparatory run, each picture is relegated a rate certainty. The certainty percent portrays the level up to which demonstrate was able to foresee the result. After the primary expectation run, the names are appeared, sorted in arrange of certainty. Convolution Neural Network is utilized to excavate the profound data of multi-layer organize within the handle of face acknowledgment. It moreover applies a channel to an input to form a highlight outline that summarizes the nearness of recognized highlights within the input. Accuracy in testing is shown in Figure 6.

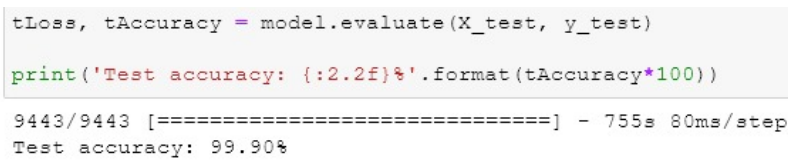

Figure 6. Test Accuracy on the Model

\section{Result and Discussion}

The outcome about this project shows that extension has more strength and exactness for detecting the American Sign Dialect. The proposed approach is being evaluated in genuine world circumstances by enforcing it through the medium of a webcam, occasionally it recognizes the problems that were not prepared to be ascertained. In this manner, it can be affirmed that this extended discovery demonstrates a solution as well. In the past, there are various issues which cause low accuracy in recognition and detection of a particular phrase or letter using various algorithms for images [18]. This might transpire scheduled to fewer components that impact the precision of the recognition such as picture clatter, the need for an assortment of diversion in the object pictures, an inadequate number of prepared images. The images are captured from the camera, which are detected and recognized as a phrase or a letter by using YOLO and CNN. Finally, they are converted to text format and displayed to ordinary individuals.

\section{Conclusion}

The proposed system contemplated an approach to develop a modified American Sign Dialect Translator which utilizing Convolutional Neural Network (along with YOLO) in a real world scenario. The proposed system is verified to detect the hand gestures at an accuracy of $92.5 \%$ for commonly used phrases. In future, a high-end semantic examination can be connected to the operative apparatus to improve the acknowledgment competency complicated individual assignments. The acknowledgment rate also can be expanded by improving the handling picture step as future work. 


\section{References}

[1] Albawi S, Mohammed TA, Al-Zawi S. Understanding of a convolutional neural network. In2017 International Conference on Engineering and Technology (ICET) 2017 Aug 21 (pp. 1-6). IEEE.

[2] Rajendran PS. Gesture Supporting Smart Notice Board Using Augmented Reality. InInternational Conference on Next Generation Computing Technologies 2017 Oct 30 (pp. 112-123). Springer, Singapore.

[3] Martin Sagayam K, Jude Hemanth D. Hand posture and gesture recognition techniques for virtual reality applications: a survey. Virtual Reality. 2017 Jun;21(2):91-107.

[4] Kumaran N, Rangaraj V, Dhanalakshmi R. Intelligent Personal Assistant-Implementing Voice Commands enabling Speech Recognition. In2020 International Conference on System, Computation, Automation and Networking (ICSCAN) 2020 Jul 3 (pp. 1-5). IEEE.

[5] Badhe PC, Kulkarni V. Indian sign language translator using gesture recognition algorithm. In2015 IEEE International Conference on Computer Graphics, Vision and Information Security (CGVIS) 2015 Nov 2 (pp. 195-200). IEEE.

[6] Lin HI, Hsu MH, Chen WK. Human hand gesture recognition using a convolution neural network. In2014 IEEE International Conference on Automation Science and Engineering (CASE) 2014 Aug 18 (pp. 1038-1043). IEEE.

[7] Karayılan T, Kılıç Ö. Sign language recognition. In2017 International Conference on Computer Science and Engineering (UBMK) 2017 Oct 5 (pp. 1122-1126). IEEE.

[8] ISL Dictionary Launch — Indian Sign Language Research and Training Center (ISLRTC), Government of India. 2019 [Internet]. Available from: http://www.islrtc.nic.in/isl-dictionary-launch.

[9] Deora D, Bajaj N. Indian sign language recognition. In2012 1st International Conference on Emerging Technology Trends in Electronics, Communication \& Networking 2012 Dec 19 (pp. 1-5). IEEE.

[10] Sarah I, Soundarya K, Dhanalakshmi R, Deenadayalan T. DYS-I-CAN: An Aid for the Dyslexic to improve the skills using Mobile Application. In2020 International Conference on System, Computation, Automation and Networking (ICSCAN) 2020 Jul 3 (pp. 1-5). IEEE.

[11] Wankhede J, Kumar M, Sambandam P. Efficient heart disease prediction-based on optimal feature selection using DFCSS and classification by improved Elman-SFO. IET Systems Biology. 2020 Sep 16;14(6):380-90.

[12] Islam MR, Mitu UK, Bhuiyan RA, Shin J. Hand gesture feature extraction using deep convolutional neural network for recognizing American sign language. In2018 4th International Conference on Frontiers of Signal Processing (ICFSP) 2018 Sep 24 (pp. 115-119). IEEE.

[13] Belagiannis V, Zisserman A. Recurrent human pose estimation. In2017 12th IEEE International Conference on Automatic Face \& Gesture Recognition (FG 2017) 2017 May 30 (pp. 468-475). IEEE.

[14] Martin Sagayam K, Jude Hemanth D. ABC algorithm based optimization of 1-D hidden Markov model for hand gesture recognition applications. Computers in Industry. 2018 Aug 1;99:313-23.

[15] Martin Sagayam K, Jude Hemanth D. A probabilistic model for state sequence analysis in hidden Markov model for hand gesture recognition. Computational Intelligence. 2019 Feb;35(1):59-81.

[16] Estrada Jiménez LA, Benalcázar ME, Sotomayor N. Gesture recognition and machine learning applied to sign language translation. InVII Latin American Congress on Biomedical Engineering CLAIB 2016, Bucaramanga, Santander, Colombia, October 26th-28th, 20162017 (pp. 233-236). Springer, Singapore.

[17] Prakash A, Krishnaveni R, Dhanalakshmi R. Continuous user authentication using multimodal biometric traits with optimal feature level fusion. International Journal of Biomedical Engineering and Technology. 2020;34(1):1-9.

[18] Kumaresan J, Perinbam JR, Ebenezer D, Vasanthi R. IRIS recognition optimized by ICA using parallel CAT swarm optimization. Journal of Engineering and Applied Sciences. 2015;10:4942-7. 